\title{
Vulnerabilidade e estratégias de adesão ao tratamento da tuberculose: discurso dos enfermeiros da atenção primária
}

Vulnerability and strategies for adherence to tuberculosis treatment: primary care nurses' discourse Vulnerabilidad y estrategias de adhesión al tratamiento de la tuberculosis discurso de los enfermeros de la atención primaria

\section{Jonh Jorge Costa Barros ${ }^{\mathrm{I}}$, Aliéren Honório Oliveira ${ }^{\mathrm{II}}$, Jeane Lima Cavalcante ${ }^{\mathrm{III}}$, Tacyla Geyce Freire Muniz Januário ${ }^{\mathrm{Iv}}$, Maria Lúcia Duarte Pereirav, Edilma Gomes Rocha Cavalcante ${ }^{\mathrm{VI}}$}

\begin{abstract}
Resumo: Objetivo: identificar as estratégias dos enfermeiros para potencializar a adesão de pacientes em tratamento de tuberculose diante de suas vulnerabilidades ao abandono. Método: estudo descritivo de abordagem qualitativa, realizado com 13 enfermeiros da Atenção Primária à Saúde de um município do Ceará, Brasil. Utilizouse entrevista semiestrutura que foram submetidas à técnica do Discurso do Sujeito Coletivo. Resultado: as vulnerabilidades ao abandono foram: condições de saúde; aspectos comportamentais; falta de moradia, dinheiro e apoio familiar e falta de incentivo dos profissionais de saúde. As estratégias para potencializar a adesão forma: educação em saúde; acolhimento e corresponsabilidade; busca de faltosos, monitoramento de exames e da adesão ao tratamento e, interdisciplinaridade e intersetorialidade. Conclusão: os enfermeiros utilizaram estratégias para potencializar a adesão ao tratamento com foco nas dimensões assistências e educacionais diante às vulnerabilidades dos pacientes. Necessitando ampliar a estratégia do tratamento diretamente observado para redução do abandono.
\end{abstract}

Descritores: Tuberculose; Adesão à medicação; Enfermagem em saúde comunitária; Atenção primária à saúde; Cuidados de enfermagem

Abstract: Objective: to identify nurses' strategies to enhance adherence of patients on tuberculosis treatment in

\footnotetext{
${ }^{\text {I }}$ Enfermeiro, Especialista em Saúde da Família pela Universidade Regional do Cariri, Crato, CE, Brasil. jonhne.10@hotmail.com, Orcid: https://orcid.org/0000-0002-7484-157X

II Enfermeira. Mestre em Enfermagem. Enfermeira da Secretaria de Saúde do Município de Farias Brito, CE, Brasil. alierenoliveira@hotmail.com, Orcid: http://orcid.org/0000-0003-4702-3691

III Enfermeira. Mestre em Enfermagem. E-mail: jeanecavalcante2009@hotmail.com, Orcid: https://orcid.org/0000-0001-9074-8263

IV Discente do curso de Enfermagem da Universidade Regional do Cariri, Crato, CE, Brasil. tacyla_@hotmail.com, Orcid: https://orcid.org/00000002-4946-9382.

V Enfermeira. Doutora. Docente do Departamento de Enfermagem da Universidade Estadual do Ceará, Fortaleza, CE, Brasil. luciad029@gmail.com, Orcid: http://orcid.org/0000-0002-7685-6169.

VI Enfermeira. Doutora em Ciência. Docente do Departamento de Enfermagem da Universidade Regional do Cariri, Programa de PósGraduação em Enfermagem, Crato, CE, Brasil. edilma.rocha@yahoo.com.br, Orcid: http://orcid.org/0000-0002-6861-2383
} 
the face of their vulnerabilities to dropout. Method: a descriptive study of qualitative approach, carried out with 13 nurses from Primary Health Care in a municipality of Ceará, Brazil. Semi-structured interviews were used and submitted to the Collective Subject Discourse technique. Result: the vulnerabilities to dropout were: health conditions; behavioral aspects; lack of housing, money, and family support; and lack of encouragement from health professionals. The strategies to enhance adherence were: health education; welcoming and co-responsibility; search for absentees, monitoring of tests and treatment adherence, and interdisciplinarity and intersectoriality. Conclusion: the nurses used strategies to enhance treatment adherence focusing on the care and educational dimensions in face of the patients' vulnerabilities. Need to expand the strategy of directly observed treatment to reduce dropout.

Descriptors: Tuberculosis; Medication adherence; Community health nursing; Primary health care; Nursing care

Resumen: Objetivo: identificar estrategias de enfermeros para potencializar la adhesión de pacientes en tratamiento de tuberculosis delante de sus vulnerabilidades al abandono. Método: estudio descriptivo de abordaje cualitativo, realizado con 13 enfermeros de la Atención Primaria de Salud de un municipio Cearense, Brasil. Utilizado entrevista semiestructurada que fueron sometidas a técnica del Discurso del Sujeto Colectivo. Resultado: las vulnerabilidades al abandono fueron: condiciones de salud; aspectos comportamentales; falta de vivienda, dinero y apoyo familiar y falta de incentivo de profesionales de salud. Las estrategias para potencializar la adhesión forma: educación en salud; hospitalidad y corresponsabilidad; búsqueda de faltosos, monitoreo de exámenes y adhesión al tratamiento y, interdisciplinariedad e intersectorialidad. Conclusión: los enfermeros utilizaron estrategias para potencializar la adhesión al tratamiento con enfoque en las dimensiones asistenciales y educacionales delante a las vulnerabilidades de los pacientes. Necesitando ampliar la estrategia del tratamiento directamente observado para reducción del abandono.

Descriptores: Tuberculosis; Cumplimiento de la Medicación; Enfermería en Salud Comunitaria; Atención Primaria de Salud; Atención de Enfermería

\section{Introdução}

No Brasil, a tuberculose (TB) é uma doença que necessita de controle, sendo preconizado pela Organização Mundial de saúde, em 2015, que estabeleceu cura deveria ser maior ou igual a 85\% e abandono ser menor que $5 \%$, contudo seus percentuais ainda não foram alcançados. ${ }^{1}$ Esse último é influenciado por diversos fatores como a falta de informação do paciente e da família sobre a doença; o uso de álcool e outras drogas; baixa escolaridade; as barreiras sociais, econômicas, demográficas e culturais; além dos problemas inerentes ao medicamento como as reações adversas. ${ }^{2}$

O Plano Nacional pelo Fim da TB apresenta meios para potencializar o processo de adesão, entre eles: o Tratamento Diretamente Observado (TDO) voltado para fortalecer a atenção às pessoas com a doença e a divulgação de estratégias inovadoras e exitosas 
3 | Barros JJC, Oliveira AH, Cavalcante JL, Januário TGFM, Pereira MLD, Cavalcante EGR

desenvolvidas no país. ${ }^{3}$

Na literatura científica identificam-se ações de promoção de adesão ao tratamento da TB, tais como: acompanhamento da equipe de enfermagem por meio do TDO; vínculo com a equipe de saúde; apoio à adesão oferecida por equipe multidisciplinar; educação e aconselhamento; sistemas de lembretes, acordos escritos ou verbais; encorajamento para continuar o tratamento, incentivos e subsídios sociais ao paciente, e apoio social oferecido aos membros da família. ${ }^{1-4}$

Essas podem responder aos aspectos de vulnerabilidades ao abandono que vivenciam as pessoas afetadas pela TB. O conceito de vulnerabilidade divide-se em três dimensões: a individual - compreende os aspectos biológicos, comportamentais e afetivos, ancorados em relacionamentos intersubjetivos; o social - inclui questões culturais, sociais e econômicas vinculados ao gênero, étnico/raciais, crenças religiosas, exclusão social, que podem influenciar as oportunidades de acesso a bens e serviços e, a programática - analisa o modo que as políticas, programas, serviços e ações influenciam situações de vulnerabilidade. ${ }^{5-6}$

Essas dimensões possibilitam que os profissionais de saúde identifiquem as limitações e adotem medidas direcionadas para sanar o problema e promover a adesão ao tratamento da TB. ${ }^{7}$ Assim, considerando esses aspectos dos cuidados dos enfermeiros da atenção primária à saúde, o estudo objetivou identificar as estratégias dos enfermeiros para potencializar a adesão de pacientes em tratamento de TB diante de suas vulnerabilidades ao abandono.

\section{Método}

Trata-se de um estudo descritivo de abordagem qualitativa. Realizou-se em um Centro de especialidade e unidades de saúdes de dois municípios da região Sul do Ceará, Brasil, que acompanham pacientes com TB.

Os participantes do estudo foram 13 enfermeiros da Estratégia de Saúde da Família e Centro de especialidade localizados em Crato, na região Sul do Ceará. O município contava com 
25 Unidades básicas de saúde (UBS) e 66 profissionais de enfermagem na atenção básica. Quanto ao serviço de referência, as pessoas afetadas pela TB e hanseníase recebiam assistência de médico, enfermeiro e técnicos, dentre outros profissionais da saúde.

A inclusão dos participantes atendeu aos seguintes critérios de inclusão: ser enfermeiro, acompanhar pacientes em tratamento de $\mathrm{TB}$ e trabalhar na área há no mínimo três meses. Foram critérios de exclusão: afastamento do participante das atividades laborais no período da coleta de dados, devido às férias, licença maternidade e/ou tratamento de saúde, ou não estar presente na unidade após três tentativas de contato pelo pesquisador. Não houve exclusão ou recusa dos participantes elegíveis durante a pesquisa.

A coleta de dados aconteceu por meio de entrevista semiestruturada, realizadas no local de trabalho dos enfermeiros, onde foram abordados cara a cara. $\mathrm{Na}$ ocasião foi explanado o objetivo da pesquisa e, após assinaram o termo de consentimento livre e esclarecido. A entrevista foi realizada por dois acadêmicos de enfermagem, teve duração média de 15 minutos e contava com questões referentes às vulnerabilidades do abandono entendidas pelos enfermeiros e quais as estratégias que utilizavam para potencializar a adesão ao tratamento da TB. O áudio foi gravado e transcrito na integra no Software Microsoft office Word $2013^{\circledast}$. A coleta ocorreu entre dezembro de 2014 a março de 2015 com todos os enfermeiros que acompanhavam as pessoas com TB.

Para análise das falas utilizou-se o Discurso do Sujeito Coletivo (DSC), que é compreendido como uma técnica de processamento de depoimentos que expressa diretamente à representação social de determinado sujeito coletivo, por meio de discursos únicos redigidos na primeira pessoa do singular. Os recortes de falas significativas identificam as ideias centrais (ICs), as expressões chaves (ECH) e a ancoragem que se constituem de palavras ou expressões linguísticas e revelam, de maneira precisa e sintética, o sentido presente nos depoimentos. Assim, para a construção do DSC identifica-se as ICs e com base nas ECHs, buscam-se as que 
5 | Barros JJC, Oliveira AH, Cavalcante JL, Januário TGFM, Pereira MLD, Cavalcante EGR

apresentarem o mesmo sentido, sentido equivalente ou sentido complementar. ${ }^{8}$

Posteriormente, realizou-se análise conceito de vulnerabilidade, ${ }^{5-6}$ para isso tomou-se os elementos que se aproximassem das dimensões individuais, sociais e programáticas, as quais foram apreendidas pelas ICs dos DSC apresentadas nos quadros.

A pesquisa atendeu às normas da Resolução 466/2012, que norteiam pesquisas com seres humanos e teve aprovação do Comitê de Ética e Pesquisa da Universidade Regional do Cariri, obtendo parecer favorável, em 09 de dezembro de 2014, com no 904.559. Certificado de Apresentação Ética no 20689113.5.0000.5055.

\section{Resultados}

O perfil sociodemográfico dos enfermeiros mostrou predominância do sexo feminino, com idade entre 20 e 60 anos e tempo de trabalho de 1 a 10 anos. As ICs formaram os sentidos das ECH e foram agrupadas em duas categorias, apresentadas a seguir com seus DSC.

\section{Concepção de vulnerabilidades ao abandono do tratamento de tuberculose}

No Quadro 1, tem-se a concepção dos enfermeiros sobre a vulnerabilidade ao abandono do tratamento das pessoas com TB. Referente às condições de saúde e aos aspectos comportamentais do paciente, apontaram elementos de vulnerabilidade individual tais como a idade, escolaridade, ter condições crônicas (diabete e o alcoolismo); limitado conhecimento sobre a transmissão da doença e do tratamento que repercutem na adesão e autocuidado.

A necessidade de moradia, dinheiro e apoio familiar foram relacionadas aos elementos da vulnerabilidade social. Assim destacaram os aspectos socioeconômicos e ambientes que interferem na autoestima do paciente. Relativo à falta de incentivo dos profissionais de saúde, as enfermeiras apontaram aspectos da vulnerabilidade programática ao reconhecerem a necessidade de incentivar os pacientes; as dificuldades em realização a supervisão e a falha no 
retorno dos pacientes ao serviço de saúde que podem comprometer a adesão ao tratamento.

Quadro 1- Compreensão e ideia central das vulnerabilidades ao abandono do tratamento de tuberculose segundo os enfermeiros, Juazeiro do Norte, Crato, Ceará, Brasil 2015.

\begin{tabular}{|l|l|}
\hline \multicolumn{1}{|c|}{ Questão1: como você compreende a vulnerabilidade ao abandono do tratamento da TB pelos pacientes? } \\
\hline $\begin{array}{l}\text { Condições de saúde do } \\
\text { paciente e aspectos } \\
\text { comportamentais }\end{array}$ & $\begin{array}{l}\text { Ser vulnerável ao tratamento é o paciente que vive sozinho, menor nível de escolaridade; } \\
\text { outras doenças como: hipertensão, diabetes, alcoolismo e idade avançada. Quando você } \\
\text { verifica, (Eles) não estão fazendo uso do medicamento corretamente. São alcoólatras, usam } \\
\text { drogas ou são mais jovens, com alimentação inadequada. Falta de consciência sobre o } \\
\text { tratamento e autocuidado torna o paciente vulnerável. Não consegue compreender a } \\
\text { gravidade da transmissão ou ele já perdeu a vontade de viver, tem problemas psicológicos. } \\
\text { Não aceitam que tem a doença. (DSC1) }\end{array}$ \\
\hline $\begin{array}{l}\text { Necessidade de moradia, } \\
\text { dinheiro e apoio familiar }\end{array}$ & $\begin{array}{l}\text { A condição socioeconômica, ter baixa renda, falta de estabilidade financeira, de trabalho e } \\
\text { da extrema pobreza. A condição de vida, a comunidade, a insalubridade e a falta de apoio } \\
\text { da família. Quando ele não tem um ambiente propício para realizar o tratamento se trona } \\
\text { vulnerável a abandonar. O andarilho que não tem residência fixa e a pessoa que vive } \\
\text { sozinha e não receber cesta básica são difíceis de aderirem ao tratamento, pois a } \\
\text { autoestima delas está muito baixa. (DSC2) }\end{array}$ \\
\hline Falta de incentivo dos \\
profissionais de saúde
\end{tabular} \begin{tabular}{l}
$\begin{array}{l}\text { Falta de incentivo pelos próprios profissionais de saúde, tem que ficar em cima mesmo se } \\
\text { não eles abandonam, não quer vi até a unidade de saúde. Agente não tem como fazer a } \\
\text { supervisão, então confia no membro da família. (DSC3) }\end{array}$ \\
\hline
\end{tabular}

\section{Estratégias utilizadas pelos enfermeiros para potencializar a adesão ao tratamento}

As estratégias relatadas pelas enfermeiras para potencializar a adesão ao tratamento da TB tiveram como foco: a educação em saúde realizada por meio de palestra com explanação sobre a doença, transmissão e adesão ao tratamento. Quanto ao acolhimento e a corresponsabilidade no tratamento, os enfermeiros buscaram estabelecer confiança estreitando o vínculo com o paciente e envolver a família pela supervisão da terapia.

No tocante a interdisciplinaridade e a intersetorialidade, os enfermeiros buscaram responder às condições socioeconômicas e de saúde dos pacientes com TB, especialmente, relacionadas às questões alimentares e de suas condições psicológicas. Assim envolvem o Núcleo de Apoio a Saúde da Família (NASF), a assistente social e profissional especialista.

Quanto à busca de faltoso, os enfermeiros relataram que ocorre por meio de visitas 
domiciliares, contato telefônico e cartas de convocação. Além de articular com a família na busca de apoio e monitoramento da adesão ao tratamento. Contava com uma estruturação para manter a acessibilidade dos pacientes, garantir a solicitação dos exames e verificação do peso mensal. Forneciam incentivos verbais quanto ao cumprimento no retorno às consultas e a adesão ao tratamento e acompanhamento por meio do TDO.

Quadro 2 - Estratégias utilizadas pelos enfermeiros para potencializar a adesão ao tratamento segundo os participantes, Juazeiro do Norte, Crato, Ceará, Brasil, 2015.

Questão 2: quais estratégias você utiliza para potencializar a adesão ao tratamento da TB em pacientes vulneráveis?

\begin{tabular}{|c|c|}
\hline Ideia central & DSC \\
\hline Educação em saúde & $\begin{array}{l}\text { Na unidade de saúde, a gente sempre procura fazer palestras, explicando o que é } \\
\text { TB, como se transmite, a importância do tratamento e as consequências se não fizer } \\
\text { o tratamento. Deixo claro todas as informações possíveis, que a doença é curável, os } \\
\text { remédios precisam ser tomados. (DSC4) }\end{array}$ \\
\hline $\begin{array}{l}\text { Acolhimento e } \\
\text { corresponsabilidades }\end{array}$ & $\begin{array}{l}\text { Eu busco a estratégia de esclarecimento e confiança com o paciente. Explicar todo o } \\
\text { processo (da doença) e coloco a responsabilidade do tratamento também nas mãos } \\
\text { dele e da família. Que estarei com ele até o final do tratamento. A nossa maior } \\
\text { estratégia é interagir com a família, com o paciente em lócus. Conhecer toda a } \\
\text { problemática em que ele está inserido para poder tratar; procurar meios de resolver } \\
\text { aquela situação e dou o apoio, se precisa. (DSC5) }\end{array}$ \\
\hline Busca de faltosos & $\begin{array}{l}\text { Faltou alguém, a gente tem que ligar. Quando é próximo (da UBS) mando um dos } \\
\text { funcionários com uma carta convocando. Se o paciente não vem, então faço a visita } \\
\text { domiciliar. É insistir para saber por que ele faltou. A nossa taxa de abandono é zero. } \\
\text { Levo o médico, peço às vezes até para internar a pessoa para fazer o exame no } \\
\text { hospital. Peço para o agente de saúde ir lá todo dia (segunda a sexta). Eu converso } \\
\text { com o paciente, com os familiares, peço para os familiares apoiarem. (DSC7) }\end{array}$ \\
\hline $\begin{array}{l}\text { Monitoramento de } \\
\text { exames e da adesão ao } \\
\text { tratamento }\end{array}$ & $\begin{array}{l}\text { Eu não os deixo esperando lá fora por muito tempo, sempre incentivo e parabenizo } \\
\text { quando eles vêm certinhos nas datas para a UBS, tomam a medicação. Faço a } \\
\text { solicitação dos exames laboratoriais para acompanhar o tratamento e a evolução do } \\
\text { paciente, monitoro o peso. Fazemos o TDO e a agente de saúde vai todo dia à } \\
\text { residência do paciente verificar se ele está tomando a medicação, registra e olha na } \\
\text { cartela quantos comprimidos tem. Às vezes eu utilizo o argumento da bolsa família, } \\
\text { cobrando que ela tome a medicação. (DSC8) }\end{array}$ \\
\hline $\begin{array}{l}\text { Interdisciplinaridade } \\
\text { e Intersetorialidade }\end{array}$ & $\begin{array}{l}\text { Quando o paciente é baixa renda e não tem renda para se alimentar e alimentar sua } \\
\text { família, vamos até em busca de assistente social para ver a questão de cesta básica. } \\
\text { A depender do paciente, a gente trabalha o enfermeiro, médico e o Nasf. Contamos } \\
\text { com o apoio da assistente social, assim com a intervenção da psicóloga que nos } \\
\text { ajuda a melhorar esta adesão do paciente. Solicito ajuda do médico e do serviço de } \\
\text { referência que a gente trabalha em parceria. (DSC6) }\end{array}$ \\
\hline
\end{tabular}




\section{Discussão}

$\mathrm{Na}$ identificação das estratégias de enfermeiros para melhorar a adesão ao paciente em tratamento da TB, inicialmente identificou a concepção desses sobre a vulnerabilidade ao abandono que relataram elementos das dimensões individual, social e programática. Assim apontam caminhos para reiterar as práticas que corroborem com a adesão por meio de intervenções e melhor qualidade da assistência.

Os enfermeiros discorreram sobre a dimensão individual da vulnerabilidade, em que apontaram a realidade das condições de vida dos pacientes; idade; escolaridade; comorbidades; uso de álcool/droga e falta de acesso à informação sobre a doença (capacidade cognitiva). Verifica-se que há elementos que se concatenam com a necessidade de acesso de recursos que permitam a mudança de comportamento (vulnerabilidade social) das pessoas com TB.

Estudo realizado em São Paulo, Brasil, aponta que o foco nas dimensões individuais (condições de vida) e sociais (trabalho) da vulnerabilidade afeta o processo de adesão dos pacientes ao tratamento da TB. $^{7}$ Essas condições de desigualdades socioeconômica e educacional que demandam de estratégias para o seu enfrentamento, compromisso dos profissionais e o desenvolvimento de políticas de saúde que modifiquem os contextos vivenciados por esses pacientes.

Quanto aos aspectos da vulnerabilidade social, no presente estudo, os enfermeiros associaram a situação de vida da pessoa doente diante de suas condições de extrema pobreza, necessidade de moradia, dinheiro e apoio familiar que interferem no adoecimento e na adesão ao tratamento. De fato, a complexidade do cuidado na busca de apoio social demanda mobilização de atores sociais e profissionais de saúde para apoiar o cuiado e a autonomia. ${ }^{9}$

Os enfermeiros foram enfáticos quanto aos aspectos da vulnerabilidade programática, pois incluíram o próprio processo de trabalho, que requer maior desempenho da equipe de 
9 | Barros JJC, Oliveira AH, Cavalcante JL, Januário TGFM, Pereira MLD, Cavalcante EGR

saúde em relação à abordagem da adesão; programação do retorno do paciente; incorporação do TDO no cotidiano das equipes e a necessidade de disponibilizar cestas básicas. De fato, as intervenções que atendam as necessidades de saúde das pessoas com TB, devem garantir o enfrentamento das desigualdades sociais e buscar a proteção aos direitos humanos. ${ }^{10}$

Os elementos relacionados à vulnerabilidade programática retratam as deficiências nos sistemas de saúde, pois ainda não são possíveis no Sistema Único de Saúde os direitos a universalidade e a equidade na atenção à saúde das pessoas com TB pelas deficiências do setor saúde e da falta de proteção social. ${ }^{11}$ Nesse sentido, os enfermeiros devem minimizar a vulnerabilidade programática por meio de uma proposta fundamentada no cuidado integral e humanizado, que atendam às demandas das pessoas com TB, na perspectiva de acompanhá-las de acordo com a expressão de suas desigualdades sociais.

Referente às estratégias da enfermagem para potencializar a adesão ao tratamento da TB, os profissionais se utilizaram da educação em saúde, para garantir o acesso à informação sobre a doença e o tratamento, por meio de palestra ou abordagem individual nas consultas. Estudos apontam que as práticas de educação em saúde nas unidades ainda são incipientes, pois podem persiste a desinformação sobre o conceito da doença, com desigualdades quanto ao acesso aos meios de informação que interferem na dinâmica indivíduo/sociedade.12

Na prática da equipe de saúde, o acesso à informação deve ser incentivado por meio de uma abordagem dialógica, para proporcionar espaço que facilite a compreensão sobre a doença e o tratamento. Essa sensibilização corrobora com a corresponsabilidade do cuidado e, consequentemente, na incorporação de práticas de autocuidado a ser realizada pelo paciente e família, potencializado na consulta de enfermagem por meio do bom relacionamento, confiança e atenção à saúde ao longo do tratamento. ${ }^{13}$

Relativo ao acolhimento e a corresponsabilidade, os enfermeiros elencaram que buscaram garantir o vínculo, a confiança e a integração com o paciente-família quanto à responsabilidade 
do cuidado apoiado durante o processo de tratamento. Estudo realizado em Ribeirão Preto, São Paulo, Brasil, aponta que a corresponsabilidade e o acolhimento humanizado favorecem a adesão ao tratamento da TB e potencializa o vínculo com a equipe de saúde, por sua vez corrobora para que o paciente possa ser protagonista do seu tratamento. ${ }^{14}$

Outras estratégias utilizadas pelos enfermeiros foram a busca de faltoso; o monitoramento de exames e da adesão ao tratamento realizado, principalmente, por meio de visitas domiciliares. As equipes que se utilizaram o TDO recorreram à participação de agentes comunitários de saúde, para acompanhar o paciente, além de contar muitas vezes com o apoio dos familiares para apoiar o tratamento dos pacientes.

No que se refere, a modalidade do TDO, trata-se de uma estratégia que não pode ser executada apenas pela equipe de saúde da família, mas pode ser construída pelo apoio matricial envolvendo profissionais especializados e da vigilância à saúde. ${ }^{15}$ A efetivação desse monitoramento nas unidades de saúde precisa de suporte organizacional e de transporte das equipes, condições que ainda apontam baixa cobertura desse indicador quando se relaciona à busca ativa e vigilância de contato intradomiciliar de caso novos de TB. ${ }^{16}$ Diante do exposto, percebe-se que as enfermeiras têm se utilizado de estratégias para busca ativa de faltosos, mas necessitam envolver os pacientes/familiares nas consultas de acompanhamento e incorporar o TDO como apoio da gestão nas atividades cotidianas da atenção primária à saúde.

Quanto ao TDO os enfermeiros buscavam estreitar a adesão ao tratamento pelos pacientes com o recebimento do benefício do programa bolsa família. Em relação ao monitoramento de exames e da adesão ao tratamento, os enfermeiros relataram incentivar os pacientes a retornarem à unidade e a seguir corretamente a tomada da medicação. Na oportunidade solicitavam os exames de rotina, realizavam a pesagem do paciente.

Diante o exposto, identifica-se que a substancial atuação do enfermeiro por meio de ação que favoreçam a adesão tratamento da TB pelo paciente sendo necessário aprender os contextos de 
11 | Barros JJC, Oliveira AH, Cavalcante JL, Januário TGFM, Pereira MLD, Cavalcante EGR

vulnerabilidades. Uma vez essas pessoas têm, em sua maioria, necessidades básicas de alimentação e moradia. ${ }^{13}$ Essas necessidades de saúde podem ser percebidas ainda durante o itinerário terapêutico, que demandam de vinculo e apoio dos profissionais de saúde para garantia da continuidade da atenção. ${ }^{17}$ Nesse sentido, as tecnologias do contexto podem favorecer a práxis e incentivar a adesão por meio de estratégias que sejam adequadas à realidade dos serviços disponíveis. ${ }^{18}$

As ações de interdisciplinaridade e intersetorialidade foram estratégias apontadas pelos enfermeiros do presente estudo, como necessidade de atender às demandas requeridas durante o cuidado do paciente/família. Nesse sentido buscaram se articular com outros profissionais da equipe, do NASF; do serviço de assistência social e do serviço de referências e contra referência, nos quais foram incluídos os psicólogos. Essa percepção dos enfermeiros corrobora como o encontrado no município de Porto Alegre no Rio Grande do Sul, em que a responsabilidade da adesão ao tratamento da TB demanda de ações intersetoriais, na perspectiva de construção de uma rede de cuidado para atender as demandas sociais e proteção das pessoas a doença. ${ }^{19}$

Estudo, em uma capital do Nordeste brasileiro, aponta que as peculiaridades do cuidado a pessoa com TB perpassa pelo ético-profissional, o institucional-político e o social, embasados nesse compromisso os profissionais dos serviços de saúde devem garantir o acesso e a qualidade do cuidado a esses pacientes. ${ }^{20} \mathrm{Na}$ prática, esses profissionais precisam estar sensíveis e comprometidos para assegurar o acesso dos pacientes e atender as suas demandas como possibilidade de diminuir o abandono do tratamento de TB.

Como limitação do estudo, pode-se considerar o fato de o mesmo ser realizado apenas com os enfermeiros da atenção primária à saúde, de modo que não pode ser generalizado. Ressalta-se a necessidade de novos estudos em outras realidades, assim como a inclusão de pessoas com TB.

Referente à prática, pontua-se que os enfermeiros podem potencializar a adesão ao tratamento quando reconhecem as vulnerabilidades das pessoas afetadas pela TB e incorporam estratégias que viabilizem a educação em saúde, desde que contemple também ação interdisciplinar. 


\section{Conclusão}

Identificou-se que as enfermeiras utilizaram estratégias para potencializar a adesão com foco nos contextos assistências e educacionais diante às vulnerabilidades ao abandono do tratamento pelos pacientes. Essas demandas foram percebidas a partir das dimensões da vulnerabilidade dos pacientes, que incluíram suas condições de saúde e aspectos comportamentais; necessidades de moraria, dinheiro e apoio familiar, assim como a própria falta de incentivo dos profissionais de saúde.

Tendo em vista esses elementos de vulnerabilidades, as estratégias estavam imbricadas em práticas de educação em saúde; acolhimento e corresponsabilidades na busca de estabelecer o vínculo com o paciente e família e assegurar a adesão ao medicamento. Incluíram a busca de faltosos por meio de visitas domiciliares; asseguraram o acesso para o monitoramento de exames e da adesão ao tratamento por meio das consultas mensais e incorporação do TDO, embora não fosse rotina em todas as equipes de saúde. Assim como buscaram desenvolver ações interdisciplinares e intersetoriais.

Ressalta-se que as estratégias utilizadas pelas enfermeiras parecem condizentes com os contextos assistenciais e educacionais para minimizar o abandono do tratamento, especialmente quando consideraram as condições de vida e trabalho da população em sua área adstrita, reiterando as ações programáticas para que pudessem responder às necessidades de saúde dos pacientes. Contudo, a incorporação do TDO no processo de trabalho das equipes de saúde ainda se encontra fragilizada pela falta de transporte ou de incentivo para que o agente comunitário de saúde possa iniciar esse processo de monitoramento na prática em seu território.

\section{Referências}

1. Beraldo AA, Andrade RLP, Orfão NH, Silva-Sobrinho RA, Pinto ESG, Wysocki AD, et al. Adherence to tuberculosis treatment in Primary Health Care: perception of patients and professionals in a large 
13 | Barros JJC, Oliveira AH, Cavalcante JL, Januário TGFM, Pereira MLD, Cavalcante EGR

municipality. Esc Anna Nery. 2017;21(4):e20170075. doi: 10.1590/2177-9465-ean-2017-0075

2. Souza ACS, Silva MLSJ, Miranda LN. Dificuldades na adesão do plano de tratamento pelo paciente com tuberculose. Cad Grad Ciênc Biol Saúde UNIT Alagoas [Internet]. 2017 [acesso em 2020 jan 05];4(2):297-312. Disponível em: https://periodicos.set.edu.br/index.php/fitsbiosaude/article/view/4560

3. Ministério da Saúde (BR). Brasil livre da tuberculose: plano nacional pelo fim da tuberculose como problema de saúde pública [Internet]. Brasília (DF): Ministério da Saúde; 2017 [acesso em 2020 jan 05]. Disponível em: http://bvsms.saude.gov.br/bvs/publicacoes/brasil_livre_tuberculose_plano_nacional.pdf

4. Orlandi GM, Pereira EG, Biagolin REM, França FOS, Bertolozzi MR. Social incentives for adherence to tuberculosis treatment. Rev Bras Enferm. 2018;72(5):1182-8. doi:10.1590/0034-7167-2017-0654

5. Ayres JRCM. Organização das ações de atenção à saúde: modelos e práticas. Saúde Soc. 2009;18(Suppl 2):11-23. doi: 10.1590/S0104-12902009000600003

6. Malagón Oviedo RA, Czeresnia D. O conceito de vulnerabilidade e seu caráter biossocial. Interface (Botucatu). 2015;19(53):237-50. doi: 10.1590/1807-57622014.0436

7. Temoteo RCA, Figueiredo TMRM, Bertolozzi MR. Vulnerabilidade individual e social na adesão ao tratamento da tuberculose: estudo descritivo. Online Braz J Nurs. 2017;16:508-11. doi: 10.17665/1676-4285.20176043

8. Lefevre F, Lefevre AM. O discurso do sujeito coletivo: um novo enfoque em pesquisa qualitativa. Caxias do Sul: EDUCS; 2003.

9. Sousa ERF, Castro KF, Cavalcante JL, Silva KN, Barbosa RS, Santos RL, et al. Social relations and social support: the experience of caring for a person with tuberculosis in a rural community. Int $\mathrm{J}$ Develop Res [Internet]. 2019 [cited 2020 Nov 01];9(11):31672-5. Available from: http://www.journalijdr.com/social-relations-and-social-support-experience-caring-person-tuberculosisrural-community

10. Maffacciolli R, Oliveira DLLC, Brand EM. Vulnerabilidade e direitos humanos na compreensão de trajetórias de internação por tuberculose. Saúde Soc. 2017;26(1):286-99. doi: 10.1590/S010412902017168038

11. Sales CMM, Nunes GF, Rogerio WP, Castro TJ, Santos BR, Maciel EL, et al. Tuberculose e a questão social: uma revisão sistemática de estudos brasileiros. Rev Bras Pesqui Saúde. 2015;17(4):156-75. doi: 10.21722/RBPS.V17I4.14342

12. Silva PLN, Oliveira MKS, Guimarães CF, Guimarães LF, Santos LR, Alves ECS. Análise do conhecimento de moradores quanto à transmissibilidade e prevenção da tuberculose: implicações biopsicossociais. J Manag Prim Health Care. 2019;10(e3):1-16. doi: 10.14295/jmphc.v10i0.515

13. Oliveira AH, Pinto AGA, Quirino GS, Cruz RSBLC, Pereira MLD, Cavalcante EGR. Necessidades de 
saúde das pessoas com tuberculose pulmonar. Rev Enferm UFSM. 2021;11(e11):1-18. doi: $10.5902 / 2179769243901$

14. Berra TZ, Bruce ATI, Alves YM, Campoy LT, Arroyo LH, Crispim JA, et al. Fatores relacionados, tendência temporal e associação espacial do abandono de tratamento para tuberculose em Ribeirão Preto-SP. Rev Eletrônica Enferm. 2020;22:58883. doi: 10.5216/ree.v22.58883

15. Junges JR, Burille A, Tedesco J. Tratamento diretamente observado da tuberculose: análise crítica da descentralização. Interface (Botucatu). 2020;24:e190160. doi: 10.1590/interface.190160

16. Clementino FS, Marcolino EC, Gomes LB, Guerreiro JV, Miranda FAN. Ações de controle da tuberculose: análise a partir do programa de melhoria do acesso e da qualidade da atenção básica. Texto Contexto Enferm. 2016;25(4):e4660015. doi: 10.1590/0104-07072016004660015

17. Oliveira AH, Pinto AGA, Lopes MSV, Figueiredo TMRM, Cavalcante EGR. Itinerário terapêutico de pessoas com tuberculose diante de suas necessidades de saúde. Esc Anna Nery. 2019; 23(3):e201900340. doi: 10.1590/2177-9465-EAN-2019-0034

18. Temoteo RCA, Carvalho JBL, Lira ALBC, Lima MA, Sousa YG. Enfermagem na adesão ao tratamento da tuberculose e tecnologias em saúde no contexto da atenção primária. Esc Anna Nery. 2019;23(3):e20180321. doi: 10.1590/2177-9465-EAN-2018-0321

19. Freire APVS, Normann KAS, Nakata PT, Cicolella DA. Percepção da enfermagem sobre a adesão e o abandono do tratamento da tuberculose. Rev Enferm UFSM. 2020;10(e37):1-18. doi: $10.5902 / 2179769239456$

20. Cavalcante EFO, Silva DMGV. Compromisso dos enfermeiros com os cuidados dos pacientes com tuberculose. Texto Contexto Enferm. 2016;25(3):e3930015. doi: 10.1590/0104-07072016003930015

Editora Científica Chefe: Cristiane Cardoso de Paula

Editora Associada: Maria Denise Schimith

Fomento: Programa Institucional de Bolsa de Iniciação Científica da Universidade Regional do Cariri (PIBIC- URCA)

\section{Autor correspondente}

Edilma Gomes Rocha Cavalcante

E-mail: edilma.rocha@yahoo.com.br

Endereço: Rua Pedro Bantin Neto, 525 Crato-CE CEP: 6310-115 
15 | Barros JJC, Oliveira AH, Cavalcante JL, Januário TGFM, Pereira MLD, Cavalcante EGR

\section{Contribuições de Autoria}

\section{1 - Jonh Jorge Costa Barro}

Concepção ou desenho do estudo/pesquisa, análise e/ou interpretação dos dados, revisão final com participação crítica e intelectual no manuscrito.

\section{2 - Aliéren Honório Oliveira}

Concepção ou desenho do estudo/pesquisa, análise e/ou interpretação dos dados, revisão final com participação crítica e intelectual no manuscrito.

\section{3 - Jeane Lima Cavalcante}

Análise e/ou interpretação dos dados, revisão final com participação crítica e intelectual no manuscrito.

\section{4 -Tacyla Geyce Freire Muniz Januário}

Análise e/ou interpretação dos dados, revisão final com participação crítica e intelectual no manuscrito.

\section{5 - Maria Lúcia Duarte Pereira}

Análise e/ou interpretação dos dados, revisão final com participação crítica e intelectual no manuscrito.

\section{6 - Edilma Gomes Rocha Cavalcante}

Concepção ou desenho do estudo/pesquisa, análise e/ou interpretação dos dados, revisão final com participação crítica e intelectual no manuscrito.

\section{Como citar este Artigo}

Barros JJC, Oliveira AH, Cavalcante JL, Januário TGFM, Pereira MLD, Cavalcante EGR. Vulnerability and strategies for adherence to tuberculosis treatment: primary care nurses' discourse. J Nurs. UFSM. 2021 [Accessed on: Year Month Day]; vol.11 e61: 1-15. DOI: https://doi.org/10.5902/2179769262654 